\title{
Prognostic Outcomes in Acute Coronary Syndrome Patients Without Standard Modifiable Risk Factors: A Multi-Ethnic Study Of 8680 Asian Patients
}

\section{Gwyneth Kong}

Yong Loo Lin School of Medicine, National University of Singapore, Singapore

Nicholas WS Chew ( $\square$ nicholas_ws_chew@nuhs.edu.sg)

Department of Cardiology, National University Heart Centre, National University Health System,

Singapore

\section{Cheng Han Ng}

Yong Loo Lin School of Medicine, National University of Singapore, Singapore

\section{Yeung Jek Ho}

Yong Loo Lin School of Medicine, National University of Singapore, Singapore

\section{Aaron ST Mai}

Yong Loo Lin School of Medicine, National University of Singapore, Singapore

Oliver ZH Lim

Yong Loo Lin School of Medicine, National University of Singapore, Singapore

\section{Anand Ambhore}

Yong Loo Lin School of Medicine, National University of Singapore, Singapore

\section{Gavin $\mathrm{Ng}$}

Yong Loo Lin School of Medicine, National University of Singapore, Singapore

William Kong

Yong Loo Lin School of Medicine, National University of Singapore, Singapore

Kian-Keong Poh

Yong Loo Lin School of Medicine, National University of Singapore, Singapore

\section{Roger Foo}

Yong Loo Lin School of Medicine, National University of Singapore, Singapore James Yip

Yong Loo Lin School of Medicine, National University of Singapore, Singapore

\section{Tiong-Cheng Yeo}

Yong Loo Lin School of Medicine, National University of Singapore, Singapore

\section{Adrian Fatt-Hoe Low}

Yong Loo Lin School of Medicine, National University of Singapore, Singapore Chi-Hang Lee

Yong Loo Lin School of Medicine, National University of Singapore, Singapore 


\section{Mark Yan-Yee Chan}

Yong Loo Lin School of Medicine, National University of Singapore, Singapore Huay-Cheem Tan

Yong Loo Lin School of Medicine, National University of Singapore, Singapore Poay-Huan Loh

Yong Loo Lin School of Medicine, National University of Singapore, Singapore

\section{Research Article}

Keywords: Acute Coronary Syndrome, standard modifiable cardiovascular risk factors, prognostic outcomes

Posted Date: September 27th, 2021

DOl: https://doi.org/10.21203/rs.3.rs-941766/v1

License: (c) (i) This work is licensed under a Creative Commons Attribution 4.0 International License. Read Full License 


\section{Abstract \\ IMPORTANCE}

There are growing concerns that patients suffering from acute coronary syndrome (ACS) without standard modifiable cardiovascular risk factors (SMuRFs), including hypertension, hypercholesterolemia, diabetes and smoking, have increased mortality.

\section{OBJECTIVE}

This study examined the outcomes of ACS in patients without SMuRFs (termed SMuRF-less) in a multiethnic Asian population which remains unknown.

\section{DESIGN}

This retrospective study was conducted from $1^{\text {st }}$ January 2011 to $31^{\text {st }}$ March 2021.

\section{SETTING}

This study was conducted in a tertiary healthcare institution in Singapore.

\section{PARTICIPANTS}

Consecutive patients presenting with ACS disease were recruited in the study.

\section{EXPOSURE}

The outcomes of SMuRF-less patients were compared with SMuRF patients.

\section{MAIN OUTCOMES AND MEASURES}

The primary outcome was cardiovascular mortality. Secondary outcomes were all-cause mortality, readmission, cardiogenic shock, stroke and heart failure. Multivariable regression analysis adjusted for covariates including age, sex, ethnicity, chronic kidney disease, ACS type, cardiac arrest, and left main and/or left anterior descending coronary

\section{RESULTS}

Of the 8680 patients studied, $8.6 \%$ were SMuRF-less patients. SMuRF-less patients were significantly younger and had fewer comorbidities including stroke and chronic kidney disease, but higher rates of ventricular arrhythmias and inotropic or invasive ventilation requirement compared to the SMuRF group. Multivariable analysis showed higher rates of cardiovascular mortality (HR 1.48, 95\% $\mathrm{Cl} 1.09-1.86$, $\mathrm{p}=0.048$ ), cardiogenic shock (RR: $1.31,95 \% \mathrm{Cl} 1.09-1.52, \mathrm{p}=0.015)$ and stroke (RR: $2.51,95 \% \mathrm{Cl} 1.67-$ 3.34, $\mathrm{p}=0.030$ ) in SMuRF-less patients compared to SMuRF patients. Both groups had similar readmission (RR: $1.10,95 \% \mathrm{Cl} 0.87-1.39, \mathrm{p}=0.413$ ) and heart failure (RR: $0.82,95 \% \mathrm{Cl} 0.56-1.21$, 
$\mathrm{p}=0.326$ ) rates. Kaplan-Meier curve showed higher 30-day cardiovascular mortality in the SMuRF-less group compared to SMuRF group (HR: 1.84, 95\% Cl 1.45-2.33, p<0.001), with similar significant trends found in men, STEMI patients, and the three Asian ethnicities.

\section{CONCLUSION AND RELEVANCE}

Although the proportion of ACS patients without standard risk factors in our Asian population is lower than those reported in the West, they also have worse short-term mortality compared to those with SMuRF. This calls for a global focus on the management of this unexpectedly high-risk subgroup of patients.

\section{Key Points}

Question: What are the outcomes of acute coronary syndrome (ACS) patients without standard modifiable risk factors (SMuRF) in a multi-ethnic Asian population?

Findings: In this cohort study of 8680 patients presenting with ACS, SMuRF-less patients made up $8.6 \%$ of the population. They had higher rates of cardiovascular mortality, stroke and cardiogenic shock compared to those with SMuRF. Excess mortality was demonstrated in SMuRF-less men, and patients with ST-segment elevation myocardial infarction.

Meaning: This calls for a global focus on the management of this unexpectedly high-risk subgroup of SMuRF-less patients.

\section{Introduction}

The control of cardiovascular risk factors is paramount in the prevention of adverse cardiovascular outcomes including stroke and acute coronary syndrome (ACS) ${ }^{1}$. Hence, early identification and intervention of standard modifiable risk factors (SMuRF), such as hypercholesterolemia ${ }^{2}$, hypertension ${ }^{3}$, smoking ${ }^{4}$ and diabetes mellitus ${ }^{5}$, are essential in reducing the atherosclerotic cardiovascular disease risk of all individuals ${ }^{6-9}$ and prevention of cardiovascular disease ${ }^{10}$. Recent studies have shown a growing proportion of patients without SMuRF (termed SMuRF-less ${ }^{11}$ ) who were previously asymptomatic, presenting with ST-segment elevation myocardial infarction (STEMI) ${ }^{12}$. Their prevalence among patients presenting with STEMI has increased over the past decade from $13 \%$ to approximately $27 \%{ }^{13,14}$, and these patients have a higher in-hospital mortality compared to patients with at least one SMuRF ${ }^{14}$.

To date, there is a paucity of studies examining the outcomes of this pragmatically challenging group of SMuRF-less patients who present with ACS. They are often overlooked in large clinical trials which rarely report the absence of standard modifiable risk factors or are less often recruited into trials targeting at atherosclerotic cardiovascular risk intervention. Despite the increasing focus on this group of patients in the West ${ }^{14-17}$, SMuRF-less cohort has not been described in Asia. In addition, most previous studies are 
only limited to the subgroup of SMuRF-less patients presenting with STEMI, with only few studies on patients with Non-STEMI (NSTEMI) ${ }^{15,18,19}$. This study will be the first to describe the prevalence and characteristic of SMuRF-less patients, and compare their outcomes to those with SMuRF in a large diverse Asian population presenting with ACS.

\section{Methods}

\subsection{Setting and Design}

Consecutive patients presenting with ACS to a major tertiary academic percutaneous coronary intervention (PCI)-capable hospital in Singapore between 1st January 2011 and 31st March 2021 were retrospectively studied. The hospital is part of the Western network that provides $\mathrm{PCl}$ services, including round-the-clock primary PCl, to the western region in Singapore ${ }^{20}$. This Western network is a hub-andspoke system that consists of our hospital (hub) and two other spoke hospitals. The patients presented with either STEMI or NSTEMI via the Emergency Department at the hub hospital or via inter-hospital transfer from the two spoke hospitals.

Patients included in the study were at least 18 years of age and presented with ACS. Patients with previous $A C S$, percutaneous coronary intervention $(P C I)$ or coronary artery bypass graft $(C A B G)$ were excluded. SMuRFs ${ }^{6,21}$ were defined as having at least one of the following cardiovascular risk factors: ex-smoker or current smoker, hypertension, diabetes mellitus, or hypercholesterolemia. Hypertension was defined as previously diagnosed hypertension, prescribed anti-hypertensives, or newly diagnosed hypertension during the index admission. Diabetes mellitus was defined as previously diagnosed type 1 or 2 diabetes, prescribed glucose lowering medications, or newly diagnosed diabetes during index admission. Hypercholesterolaemia was defined as previously diagnosed hypercholesterolaemia, prescribed lipid-lowering therapy, or newly diagnosed hypercholesterolaemia during index admission. As both fasting glucose and acute-phase blood pressure readings are influenced by neurohormonal response to acute myocardial infarction, these were not incorporated in the definitions.

Patients were allocated to 2 study groups according to their SMuRF status: (1) SMuRF, defined as having 1 or more SMuRFs, or (2) SMuRF-less, defined as the absence of SMuRF.

\subsection{Data Collection}

Data on baseline demographic and clinical characteristics, SMuRF status, previous medical history, clinical status at presentation, angiographic and procedural characteristics, echocardiographic characteristics and medications on discharge were retrospectively collected from the electronic clinical records. Information on in-hospital complications were also retrieved.

\subsection{Study Outcomes}

The follow-up period was 30 days and primary outcome was cardiovascular mortality. Secondary outcomes were in-hospital all-cause mortality, unplanned readmission, cardiogenic shock, heart failure, 
and stroke. Cardiovascular mortality was defined as any death due to any cardiovascular causes, and allcause mortality was defined as death due to any or unexplained causes. Cardiogenic shock was defined by the presence of persistent hypotension defined as SBP $<90 \mathrm{~mm} \mathrm{Hg}$ or mean arterial pressure (MAP) 30 $\mathrm{mm} \mathrm{Hg}$ below the baseline, cardiac index $(<1.8 \mathrm{~L} / \mathrm{min} / \mathrm{m} 2$ without support or $<2.2 \mathrm{~L} / \mathrm{min} / \mathrm{m} 2$ with support) with adequate or elevated filling pressures (left ventricular end diastolic pressure $>18 \mathrm{~mm} \mathrm{Hg}$ or right ventricular end diastolic pressure $>10$ to $15 \mathrm{~mm} \mathrm{Hg})^{22}$. Heart failure was defined clinically based on development of typical signs and symptoms, with structural and functional cardiac abnormalities ${ }^{23}$.

Statistical analysis was conducted on STATA 16.1 (Statacorp) and IBM SPSS Statistics 25 (SPSS Inc., Chicago, IL, USA). A p-value of $\leq 0.05$ was considered statistically significant. To compare between baseline characteristics of included patients, either chi square analysis or fisher exact test was used to compare categorical and binary variables. Unpaired t-test was used in the analysis of continuous variables. Cardiovascular mortality was assessed in the Fine-Gray model with hazard ratio (HR) to account for competing risk. The issue of competing risk has been well described by Abdel-Qadir et al. ${ }^{24}$ In the analysis of binary outcomes including 30-day myocardial infarction, stroke, cardiogenic shock, heart failure and readmission, a generalized linear regression with a log link, gaussian distribution, and robust variance estimator were used to compute the risk ratios $(\mathrm{RR})^{25}$. The risk ratio was preferred due to the ease of interpretation compared to an odds ratio ${ }^{26}$. Multivariable adjustment was performed with age, sex, ethnicity, chronic kidney disease, ACS type (STEMI and NSTEMI), cardiac arrest and presence of left main coronary and/or left anterior descending coronary artery disease included in the model. The KaplanMeier survival curves for cardiovascular mortality was constructed from the date of admission up to 30 days. The survival curves were further stratified according to the sex, the three main Asian ethnicities (Chinese, Malay and Indian), and the ACS type (STEMI and NSTEMI). The study was approved by the local institutional review committee in accordance to the revised Declaration of Helsinki (NHG Research DSRB: 2021/00089-AMD0001). As the study involved the retrospective analysis of clinically acquired data, the institutional review board waived the need for written patient consent.

\section{Results}

\subsection{Study Cohort Characteristics}

Of the 8680 patients with ACS enrolled into the study, 7934 (91.4\%) patients were in the SMuRF group and $746(8.6 \%)$ in the SMuRF-less group. The follow-up time was 30 days. Between 2011 and 2021, the yearly prevalence of SMuRF-less patients presenting with ACS fluctuated little and ranged from 5.9$10.7 \%$ (Fig. 1).

A total of 4975 (57.3\%) patients presented with STEMI and 3705 (42.7\%) with NSTEMI. A larger proportion of SMuRF-less patients presented with STEMI as compared to SMuRF patients (482 [64.5\%] versus 4493 [56.6\%] respectively, $p<0.001)$. The mean age of SMuRF-less patients was significantly lower than that of SMuRF patients ( $57 \pm 14$ years versus $61 \pm 13$ years respectively, $p<0.001$ ). SMuRF- 
less patients were also less likely to have history of stroke $(1.9 \%$ versus $6.0 \%$ respectively, $p<0.001)$ and chronic kidney disease ( $2.3 \%$ versus $10.2 \%$ respectively, $p<0.001)$, compared to SMuRF patients. In the SMuRF group, the prevalence of hypertension (60.4\%), hypercholesterolemia (58.8\%), and diabetes mellitus (41.9\%) was high. Among these SMuRF patients, 3161 (41.2\%) were current smokers, 964 (12.6\%) ex-smokers and 3548 (46.2\%) non-smokers. The baseline characteristics of the patients are outlined in Table 1.

During the index hospitalisation, SMuRF-less patients experienced higher rates of PCI (83.0\% versus $79.0 \%$ respectively, $p=0.018$ ) and were more likely to have culprit vessel involving the left anterior descending artery (56.6\% versus $50.9 \%$ respectively), or left main coronary artery (3.4\% versus $1.9 \%$ respectively) compared to SMuRF patients $(p=0.012)$. Additionally, SMuRF-less patients had higher prevalence of multivessel disease $(1.9 \%$ versus $0.9 \%$ respectively, $p=0.018)$. Both groups of patients did not differ in the number of vessels and stents involved in $\mathrm{PCl}$, post-PCl Thrombolysis in Myocardial Infarction (TIMI) flow score, PCI success rate and need for CABG.

SMuRF-less patients also had significantly higher rates of ventricular arrhythmias, and inotropic and invasive ventilation support when compared to SMuRF patients. However, ischemic mitral regurgitation was less common in the SMuRF-less than SMuRF patients. The incidences of other in-hospital complications including sepsis, atrial fibrillation, bleeding events, and acute renal failure were similar between the two groups of patients; and so was the length of hospital stay. On discharge, the SMuRF-less group was less likely to be prescribed ACE-inhibitors (ACE-I) or angiotensin-II receptor blockers (ARBs), $\beta$ blockers, and statins compared to the SMuRF group.

\subsection{Study Outcomes}

The cardiovascular mortality $(10.7 \%$ versus $6.1 \%$ respectively, $p<0.001)$, all-cause mortality $(11.7 \%$ versus $6.9 \%$ respectively, $p<0.001)$ and cardiogenic shock $(16.3 \%$ versus $8.6 \%$ respectively, $p<0.001)$ was significantly higher in SMuRF-less patients compared to SMuRF patients. The incidences of stroke, hospital readmission and heart failure were similar between both study groups.

The Kaplan-Meier curve of 30-day cardiovascular mortality are presented based on study cohort, sex, ACS type and ethnicity (Fig. 2). For the overall study cohort, the cumulative event curves diverged early from the day of ACS presentation indicating higher early mortality in the SMuRF-less group compared to the SMuRF group which was sustained over the 30-day follow-up period (HR 1.837, 95\% Cl 1.450-2.328, p < 0.001). Similar trend was found in the men (HR 2.043, 95\% Cl 1.557-2.680, p < 0.001), but not for women (HR 1.390, 95\% Cl 0.852-2.270, p =0.187). For each of the ethnicities, such trend was observed in the Chinese (HR 1.672, 95\% Cl 1.222-2.289, p=0.001), Malay (HR 2.904, 95\% Cl 1.656-5.094, p<0.001) and Indian (HR 2.055, 95\% Cl 1.185-3.563, $\mathrm{p}=0.010)$. This trend was also observed in the patients with STEMI (HR 1.947, 95\% Cl 1.501-2.527, p < 0.001) but not in patients with NSTEMI (HR 1.188, 95\% Cl $0.658-2.145, p=0.567)$. 
The multivariable analysis showed that SMuRF-less patients had higher risk of cardiovascular mortality (HR 1.48, 95\% Cl 1.09-1.86, $p=0.048$ ), cardiogenic shock (RR: 1.31, 95\% $\mathrm{Cl} 1.09-1.52, \mathrm{p}=0.015$ ) and stroke (RR: $2.51,95 \% \mathrm{Cl} 1.67-3.34, \mathrm{p}=0.030$ ) compared to the SMuRF patients despite adjusting for important confounders (Fig. 3). The risk of unplanned readmission (RR: 1.10,95\% Cl 0.87- 1.39, $p=$ 0.413 ) and heart failure (RR: $0.82,95 \% \mathrm{Cl} 0.56-1.21, \mathrm{p}=0.326$ ) was similar between both patient groups.

\section{Discussion}

This study is the first to examine the prognostic outcomes of an often-overlooked subset of patients without standard modifiable risk factors, in a typically understudied Asian population presenting with ACS. It is also the first of such study to include both patients with NSTEMI and STEMI. The main findings of the study are: 1) The prevalence of SMuRF-less patients presenting with ACS in an Asian cohort was $8.6 \%$, with its yearly prevalence relatively constant over the past decade; 2) SMuRF-less patients tend to present in a more critical state compared to SMuRF patients, with higher rates of ventricular arrhythmia, and requirement for inotropic and invasive ventilation support; 3 ) The adjusted risks of cardiovascular mortality, cardiogenic shock and stroke were significantly higher in the SMuRF-less patients compared to SMuRF patients; 4) The significantly higher cardiovascular mortality in SMuRF-less patients compared to SMuRF patients was apparent early from presentation and was sustained over the 30-day follow-up period. Such trend was observed in men and STEMI patients, but not in women or NSTEMI patients. Similar trend was also seen across all three Asian ethnicities.

Traditionally, both primary and secondary prevention of cardiovascular diseases have been focused on high risk individuals with cardiovascular risk factors ${ }^{18,27}$. As a result, the subgroup of patients without SMuRFs remains understudied. Recent studies on both STEMI and NSTEMI have demonstrated an increasing prevalence of patients without traditional risk factors ${ }^{13}$, with the prevalence of SMuRF-less patients being $10.5 \%$ in the United States of America ${ }^{19}, 14.5 \%$ in Canada ${ }^{15}, 14.9 \%$ in Sweden ${ }^{11}$ and $19-$ $25 \%$ in Australia ${ }^{13,14}$. Notably, the population of SMuRF-less patients in our Asian cohort was much lower with a prevalence of only $8.6 \%$. The stark difference in the proportion of SMuRF-less patients across the globe might be partly explained by the differences in risk factor identification ${ }^{28}$, genetic predisposition ${ }^{29}$, lifestyle factors such as smoking and physical activity ${ }^{30,31}$, and individual country's primary prevention programme ${ }^{28,32}$. Even with the presence of traditional cardiovascular risk factors, their impacts might vary across different ethnic groups, with stroke being more common among hypertensive patients in Asia and chronic heart disease more prevalent in the West ${ }^{33}$. Despite relatively lower than that seen in the West, the prevalence of SMuRF-less patients in our Asian cohort remains sizeable and warrants further attention to address specific modifiable factors that might predispose Asians to various cardiovascular comorbidities.

Even though the SMuRF-less patients in our cohort were generally younger and had fewer baseline comorbidities, their cardiovascular mortality was higher than those with conventional risk factors. This is consistent with the findings from previous studies ${ }^{11,14-16,19}$ based in the West, and could be partly 
explained by multiple postulated reasons. Several cardiovascular risk factors, such as serum cholesterol or glycated haemoglobin A1c, have a linear relationship with the risk of cardiovascular morbidity and categorising the patients into binary groups using a standard diagnostic threshold can potentially introduce selection bias by missing out on patients with borderline measurements for certain risk factor that have not reached the diagnostic thresholds. As mentioned earlier, individuals with pre-disease state for various cardiovascular risk factors might also have a higher atherosclerotic cardiovascular risk. Moreover, the role of less well established risk factors such as body mass index, triglyceride concentrations, high-density lipoprotein concentration and sedentary lifestyle, which might also be the potential drivers of atherosclerosis but have not been concomitantly evaluated. Additionally, some recognised risk factors such as abdominal obesity, psychosocial factors, sedentary lifestyle, dietary factors and alcohol consumption are not easily quantified and hence their potential impact on the outcome of SMuRF-less patients is not well assessed ${ }^{34}$. Furthermore, as patients with known risk factors are more likely to be on treatment, the ACS severity may have been modified by evidence-based therapy used in primary intervention ${ }^{16}$ leading to better outcome among the SMuRF patients.

The pathogenesis of atherosclerosis, especially its genetic basis, is also not fully understood. A recent study reported as many as 55 genetic loci that are associated with coronary artery disease, with more than $66 \%$ of them not linked to the traditional risk factors ${ }^{35}$. Compared to the patients with SMuRF, more SMuRF-less patients in our study were of Indian ethnicity and had family history of premature coronary artery disease highly suggestive of a genetic predilection to develop atherosclerotic cardiovascular disease. It is plausible that these genetic factors might play a major role in the disease process among SMuRF-less patients leading to onset of disease at a younger age and more advanced disease at presentation with consequent worse prognosis. This raises the possibility that other unknown factors might be involved in the pathogenesis and presentation of ACS among the SMuRF-less patients.

Our study found an increased short-term cardiovascular mortality only in the male SMuRF-less patients, which is in contrary to the observation by Figtree et a ${ }^{11}$ which showed that SMuRF-less women with STEMI had an excess of short-term mortality over their men counterparts. Such discrepancy in observation could be partly attributed to constitutional differences in study population including ethnic background and the inclusion of full spectrum of ACS patients in our study as opposed to the subgroup of STEMI patients in Figtree et als study. Other possible factors include varying extent of delay in patient presentation ${ }^{36}$ and hormonal-mediated differences associated with atherosclerotic plaque characteristics ${ }^{37}$. However, our study was not granular enough to explain such differences and future studies are warranted.

Moreover, significant mortality difference between our SMuRF and SMuRF-less patients was only observed in the STEMI, but not NSTEMI patients. This is in contrast to a prior study that showed increased mortality in SMuRF-less as compared to SMURF patients with NSTEMI ${ }^{19}$. One possible reason for this discrepancy is the difference in PCl rates between SMuRF and SMuRF-less patients as described by Roe et al, such that the lower use of invasive procedures in SMuRF-less patients might have 
contributed to their higher mortality ${ }^{19}$. Hence, similar PCI rates between SMuRF-less and SMuRF patients (53.2\% versus $48.9 \%$ respectively, $p=0.321)$ might partly explain the similar mortality between the two groups of NSTEMI patients. Another possible reason is the significantly lower mortality events in NSTEMI as compared to STEMI patients $(4.1 \%$ versus $8.3 \%$ respectively, $p<0.001)$ which might lead to less apparent difference seen between SMuRF and SMuRF-less patients among our NSTEMI patients ${ }^{38}$.

Similar to current literature $11,15,16$, we found that SMuRF-less patients were less likely to be treated with guideline-directed medication including beta-blockers, statins and/or ACE-I or ARBs when compared to the SMuRF patients. Figtree et a ${ }^{11}$ has shown that suboptimal prescription rate of ACE-I or ARBs and beta-blockers was directly correlated to a higher mortality among the SMuRF-less patients which is in line with other studies demonstrating the prognostic benefit of early initiation of beta-blocker and ACE-I in patients with $\mathrm{ACS}^{39-41}$. The reason SMuRF-less patients were less likely to be prescribed with prognostically important medication was unclear but could be related to the false perception that they were of lower cardiac risk. The worse clinical status at presentation, lack of pre-existing hypertension and higher incidence of stroke among the SMuRF-less patients might lead to a poorer hemodynamics which precluded the use of beta-blockers or ACE-I or ARBs. Increased awareness of the paradoxical unfavorable outcome in SMuRF-less patients presenting with ACS should be widely promoted and early initiation of guideline-directed medical therapy among ACS patients remains crucial regardless of the cardiovascular risk factor status.

\section{Clinical Implications}

Our findings raise concerns regarding the unfavourable outcome in SMuRF-less patients presenting with ACS among the Asian population. Such patients are not uncommon and may present in an even worse clinical state than those with one or more standard cardiovascular risk factors. These ameliorate the general sense of complacency that significant coronary artery disease is an unlikely health concern in individuals without cardiovascular risk factors. Clinicians need to be aware of this unexplained paradoxical phenomenon, and effective lifestyle and pharmacological intervention need to be optimised in all patients regardless of their SMuRF status. Although lower than that reported in the West, the proportion of SMuRF-less patients in our Asian population remains sizeable indicating that this is a global phenomenon that warrant its due attention by all healthcare systems. More efforts are needed to understand the underlying pathophysiology of atherosclerotic cardiovascular risk factors in SMuRF-less patients, from the onset of atherosclerosis through to its progression and the occurrence of ACS, in order to identify such individuals so that appropriate and timely preventative intervention can be given.

Currently, most published studies were limited to short-term outcomes and hence further studies are also needed in order to understand the long-term outcome of SMuRF-less patients with ACS.

\section{Strengths and Limitations}

This study is the first to examine the prognosis of SMuRF-less patients presenting with ACS in a large Asian cohort over a 10-year period. However, this study has its limitations. Firstly, this is a single-centre 
retrospective observational study which might be affected by unknown confounders and bias. Therefore, causality cannot be deduced from our results. However, such potential bias was mitigated by adjusting for important covariates in the multivariable models and using mortality as the primary study outcome. Secondly, the current method of categorizing patients into SMuRF and SMuRF-less groups might not be ideal, but it is the universal method used by all published studies, and based on local or international diagnostic threshold for each of the SMuRFs. Such thresholds are generally derived based on clinical evidence or expert consensus, and usually form the thresholds for guideline-directed treatment. Thirdly, some recognized atherosclerotic cardiovascular risk factors other than those universally considered as SMuRF are also beyond the scope for evaluation in this study. The retrospective nature of the study did not allow further evaluation of SMuRF-less patients for non-atherosclerotic cause of ACS such as protein $\mathrm{C}$ and $\mathrm{S}$ deficiency.

\section{Conclusion}

Patients presenting with ACS but without any standard modifiable risk factor are not uncommon in a multiethnic Asian population. They tend to present in a worse clinical state and have poorer short-term outcome, including higher cardiovascular mortality, compared to those with SMuRF.

\section{Declarations}

\section{Compliance with Ethical Standards:}

The authors declare that they have no conflict of interest.

\section{Funding/Support}

None reported.

\section{References}

1. Giampaoli S, Palmieri L, Mattiello A, Panico S. Definition of high risk individuals to optimise strategies for primary prevention of cardiovascular diseases. Nutrition, metabolism and cardiovascular diseases. 2005;15(1):79-85.

2. Verschuren WM, Jacobs DR, Bloemberg BP, et al. Serum total cholesterol and long-term coronary heart disease mortality in different cultures: Twenty-five-year follow-up of the seven countries study. Jama. 1995;274(2):131-136.

3. MacMahon S, Peto R, Collins R, et al. Blood pressure, stroke, and coronary heart disease: part 1, prolonged differences in blood pressure: prospective observational studies corrected for the regression dilution bias. The Lancet. 1990;335(8692):765-774.

4. Health UDo, Services H. The Health Benefits of Smoking Cessation: A Report of the Surgeon General DHHS Publication No.(CDC) 90-8416 Rockville, Maryland: US Department of Health and Human 
Services. Public Health Service, Centers for Disease Control, Center for Chronic Disease Prevention and Health Promotion, Office on Smoking and Health. 1990;

5. Stamler J, Vaccaro O, Neaton JD, Wentworth D, Group MRFITR. Diabetes, other risk factors, and 12-yr cardiovascular mortality for men screened in the Multiple Risk Factor Intervention Trial. Diabetes care. 1993;16(2):434-444.

6. TR D, FE M, GV M. Coronary heart disease in the Framingham study. American Journal of Public Health and the Nation's Health. 1957;47(4 Pt 2):4-24.

7. Kannel WB, Dawber TR, Kagan A, Revotskie N, STOKES III J. Factors of risk in the development of coronary heart disease-six-year follow-up experience: the Framingham Study. Annals of internal medicine. 1961;55(1):33-50.

8. Wilson PW, D’Agostino RB, Levy D, Belanger AM, Silbershatz H, Kannel WB. Prediction of coronary heart disease using risk factor categories. Circulation. 1998;97(18):1837-1847.

9. Ridker PM, Buring JE, Rifai N, Cook NR. Development and validation of improved algorithms for the assessment of global cardiovascular risk in women: the Reynolds Risk Score. Jama. 2007;297(6):611-619.

10. Emberson J, Whincup P, Morris R, Walker M, Ebrahim S. Evaluating the impact of population and high-risk strategies for the primary prevention of cardiovascular disease. European heart journal. 2004;25(6):484-491.

11. Figtree GA, Vernon ST, Hadziosmanovic N, et al. Mortality in STEMI patients without standard modifiable risk factors: a sex-disaggregated analysis of SWEDEHEART registry data. The Lancet. 2021;397(10279):1085-1094.

12. Piepoli MF, Hoes AW, Agewall S, et al. Guidelines: Editor's choice: 2016 European Guidelines on cardiovascular disease prevention in clinical practice: The Sixth Joint Task Force of the European Society of Cardiology and Other Societies on Cardiovascular Disease Prevention in Clinical Practice (constituted by representatives of 10 societies and by invited experts) Developed with the special contribution of the European Association for Cardiovascular Prevention \& Rehabilitation (EACPR). European Heart Journal. 2016;37(29):2315.

13. Vernon ST, Coffey S, Bhindi R, et al. Increasing proportion of ST elevation myocardial infarction patients with coronary atherosclerosis poorly explained by standard modifiable risk factors. European journal of preventive cardiology. 2017;24(17):1824-1830.

14. Vernon ST, Coffey S, D'Souza M, et al. ST-Segment-Elevation Myocardial Infarction (STEMI) Patients Without Standard Modifiable Cardiovascular Risk Factors-How Common Are They, and What Are Their Outcomes? Journal of the American Heart Association. 2019;8(21):e013296.

15. Wang JY, Goodman SG, Saltzman I, et al. Cardiovascular Risk Factors and In-hospital Mortality in Acute Coronary Syndromes: Insights From the Canadian Global Registry of Acute Coronary Events. Can J Cardiol. Dec 2015;31(12):1455-61. doi:10.1016/j.cjca.2015.04.007

16. Canto JG, Kiefe Cl, Rogers WJ, et al. Number of coronary heart disease risk factors and mortality in patients with first myocardial infarction. Jama. Nov 16 2011;306(19):2120-7. 
doi:10.1001/jama.2011.1654

17. Jernberg T, Attebring MF, Hambraeus K, et al. The Swedish Web-system for enhancement and development of evidence-based care in heart disease evaluated according to recommended therapies (SWEDEHEART). Heart. 2010;96(20):1617-1621.

18. Khot UN, Khot MB, Bajzer CT, et al. Prevalence of conventional risk factors in patients with coronary heart disease. Jama. 2003;290(7):898-904.

19. Roe MT, Halabi AR, Mehta RH, et al. Documented traditional cardiovascular risk factors and mortality in non-ST-segment elevation myocardial infarction. American heart journal. 2007;153(4):507-514.

20. Chew NW, Sia C-H, Wee H-L, et al. Impact of the COVID-19 Pandemic on Door-to-Balloon Time for Primary Percutaneous Coronary Intervention-Results From the Singapore Western STEMI Network-. Circulation Journal. 2021;85(2):139-149.

21. Piironen $\mathrm{M}$, Ukkola $\mathrm{O}$, Huikuri $\mathrm{H}$, et al. Trends in long-term prognosis after acute coronary syndrome. European journal of preventive cardiology. 2017;24(3):274-280.

22. Hochman JS, Sleeper LA, Godfrey E, et al. Should we emergently revascularize occluded coronaries for cardiogenic shock: an international randomized trial of emergency PTCA/CABG-trial design. American heart journal. 1999;137(2):313-321.

23. Ponikowski P, Voors AA, Anker SD, et al. 2016 ESC Guidelines for the diagnosis and treatment of acute and chronic heart failure: The Task Force for the diagnosis and treatment of acute and chronic heart failure of the European Society of Cardiology (ESC) Developed with the special contribution of the Heart Failure Association (HFA) of the ESC. European heart journal. 2016;37(27):2129-2200.

24. Abdel-Qadir H, Fang J, Lee DS, et al. Importance of considering competing risks in time-to-event analyses: application to stroke risk in a retrospective cohort study of elderly patients with atrial fibrillation. Circulation: Cardiovascular Quality and Outcomes. 2018;11(7):e004580.

25. Cummings P. Methods for estimating adjusted risk ratios. The Stata Journal. 2009;9(2):175-196.

26. Cummings $P$. The relative merits of risk ratios and odds ratios. Archives of pediatrics \& adolescent medicine. 2009;163(5):438-445.

27. Manuel DG, Lim J, Tanuseputro P, et al. Revisiting Rose: strategies for reducing coronary heart disease. Bmj. 2006;332(7542):659-662.

28. Perkovic V, Huxley R, Wu Y, Prabhakaran D, MacMahon S. The burden of blood pressure-related disease: a neglected priority for global health. Hypertension. 2007;50(6):991-997.

29. Cullen P, Funke H, Schulte H, Assmann G. Lipoproteins and cardiovascular risk-from genetics to CHD prevention. Journal of atherosclerosis and thrombosis. 1997;4(2):51-58.

30. Teo K, Lear S, Islam S, et al. Prevalence of a healthy lifestyle among individuals with cardiovascular disease in high-, middle-and low-income countries: The Prospective Urban Rural Epidemiology (PURE) study. Jama. 2013;309(15):1613-1621.

31. Ueshima H, Sekikawa A, Miura K, et al. Cardiovascular disease and risk factors in Asia: a selected review. Circulation. 2008;118(25):2702-2709. 
32. Mendis S, Abegunde D, Yusuf S, et al. WHO study on Prevention of REcurrences of Myocardial Infarction and StrokE (WHO-PREMISE). Bulletin of the World Health Organization. 2005;83:820-829.

33. Liu LS. [2010 Chinese guidelines for the management of hypertension]. Zhonghua Xin Xue Guan Bing Za Zhi. Jul 2011;39(7):579-615.

34. Yusuf $S$, Hawken $S$, Ôunpuu $S$, et al. Effect of potentially modifiable risk factors associated with myocardial infarction in 52 countries (the INTERHEART study): case-control study. The lancet. 2004;364(9438):937-952.

35. Deloukas P, Kanoni S, Willenborg $\mathrm{C}$, et al. Large-scale association analysis identifies new risk loci for coronary artery disease. Nature genetics. 2013;45(1):25-33.

36. van Oosterhout RE, de Boer AR, Maas AH, Rutten FH, Bots ML, Peters SA. Sex Differences in Symptom Presentation in Acute Coronary Syndromes: A Systematic Review and Meta-analysis. Journal of the American Heart Association. 2020;9(9):e014733.

37. Haider A, Bengs S, Luu J, et al. Sex and gender in cardiovascular medicine: presentation and outcomes of acute coronary syndrome. European heart journal. 2020;41(13):1328-1336.

38. Cecchi E, D'Alfonso MG, Chiostri M, et al. Impact of hypertension history on short and long-term prognosis in patients with acute myocardial infarction treated with percutaneous angioplasty: comparison between STEMI and NSTEMI. High blood pressure \& cardiovascular prevention. 2014;21(1):37-43.

39. Bugiardini R, Cenko E, Ricci B, et al. Comparison of early versus delayed oral $\beta$ blockers in acute coronary syndromes and effect on outcomes. The American journal of cardiology. 2016;117(5):760767.

40. Andersson C, Shilane D, Go AS, et al. Beta-blocker therapy and cardiac events among patients with newly diagnosed coronary heart disease. Journal of the American College of Cardiology. 2014;64(3):247-252.

41. Group I-C. ISIS-4: a randomised factorial trial assessing early oral captopril, oral mononitrate, and intravenous magnesium sulphate in 58,050 patients with suspected acute myocardial infarction. Lancet. 1995;345(8951):669-685.

\section{Table 1}

Table 1. Demographic and Clinical Characteristics of Study Cohort Presenting with Acute Coronary Syndrome, Based on Standard Modifiable Cardiovascular Risk Factors. 


\begin{tabular}{|c|c|c|c|c|}
\hline & $\begin{array}{l}\text { Overall } \\
(\mathrm{N}=8680)\end{array}$ & $\begin{array}{l}\text { SMuRF } \\
(\mathrm{N}=7934)\end{array}$ & $\begin{array}{l}\text { SMuRF-less } \\
(\mathrm{N}=746)\end{array}$ & $\begin{array}{l}\text { P- } \\
\text { Value }\end{array}$ \\
\hline Age & $61(13)$ & $61(13)$ & $57(14)$ & $<0.001$ \\
\hline Male & $6846(78.9)$ & $6254(78.8)$ & $592(79.4)$ & 0.734 \\
\hline Ethnicity & & & & $<0.001$ \\
\hline Chinese & $4889(61.0)$ & $4468(60.9)$ & $421(62.7)$ & \\
\hline Malay & $1697(21.2)$ & 1607 (21.9) & $90(13.4)$ & \\
\hline Indian & $1367(17.1)$ & $1208(16.5)$ & 159 (23.7) & \\
\hline Caucasian & $56(0.7)$ & $55(0.7)$ & $1(0.1)$ & \\
\hline Previous Stroke & $492(5.7)$ & $478(6.0)$ & $14(1.9)$ & $<0.001$ \\
\hline Atrial fibrillation & $201(2.3)$ & $189(2.4)$ & $12(1.6)$ & 0.177 \\
\hline Previous heart failure & $151(1.7)$ & $147(1.9)$ & $4(0.5)$ & 0.009 \\
\hline Chronic kidney disease & $826(9.5)$ & 809 (10.2) & $17(2.3)$ & $<0.001$ \\
\hline Family history of CAD & $928(10.7)$ & $829(10.5)$ & 99 (13.3) & 0.017 \\
\hline Diabetes Mellitus & $3324(38.3)$ & 3324 (41.9) & 0 & $<0.001$ \\
\hline Hypercholesterolemia & $4663(53.7)$ & $4663(58.8)$ & 0 & $<0.001$ \\
\hline Hypertension & $4793(55.2)$ & $4793(60.4)$ & 0 & $<0.001$ \\
\hline Smoking Status & & & & $<0.001$ \\
\hline Smoker & 3161 (37.9) & 3161 (41.2) & 0 & \\
\hline Ex-smoker & 964 (11.5) & 964 (12.6) & 0 & \\
\hline Non-smoker & $4224(50.6)$ & $3548(46.2)$ & $677(100.0)$ & \\
\hline \multicolumn{5}{|l|}{ Discharge Medication } \\
\hline$A C E-I / A R B$ & $5477(63.3)$ & $5084(64.3)$ & 393 (53.3) & $<0.001$ \\
\hline$\beta$-blocker & 6849 (79.2) & 6304 (79.7) & 545 (74.0) & $<0.001$ \\
\hline Statin & 7906 (91.3) & 7268 (91.8) & 638 (86.3) & $<0.001$ \\
\hline Aspirin & $8233(95.1)$ & 7539 (95.2) & 694 (93.9) & 0.109 \\
\hline$P 2 Y 12$ inhibitor & 6559 (95.3) & 6085 (95.3) & $574(94.6)$ & 0.405 \\
\hline Warfarin & 325 (4.7) & 291 (4.6) & 34 (5.7) & 0.239 \\
\hline Direct oral anticoagulants & $175(2.5)$ & $160(2.5)$ & $15(2.5)$ & 0.963 \\
\hline
\end{tabular}




\begin{tabular}{|c|c|c|c|c|}
\hline Peak Creatinine (mmol/L) & $129(141)$ & $130(145)$ & $108(89)$ & $<0.001$ \\
\hline Troponin I (ng/L) & $\begin{array}{l}9465 \\
(18278)\end{array}$ & 9395 (18232) & $10223(18770)$ & 0.251 \\
\hline Left ventricular ejection fraction (\%) & $49(13)$ & $49(13)$ & $49(13)$ & 0.862 \\
\hline \multicolumn{5}{|l|}{ In-hospital management } \\
\hline Presentation Route & & & & 0.867 \\
\hline Emergency Medical Services & $2436(37.3)$ & $2215(37.1)$ & $221(39.0)$ & \\
\hline Walk-in & $3024(46.3)$ & $2773(46.5)$ & $251(44.3)$ & \\
\hline Interhospital transfer & $1034(15.8)$ & $943(15.8)$ & $91(16.0)$ & \\
\hline Elective & $10(0.2)$ & $9(0.2)$ & $1(0.2)$ & \\
\hline Inpatient & $27(0.4)$ & $24(0.4)$ & $3(0.5)$ & \\
\hline ACS type & & & & $<0.001$ \\
\hline STEMI & $4975(57.3)$ & $4493(56.6)$ & $482(64.5)$ & \\
\hline NSTEMI & $3705(42.7)$ & $3441(43.4)$ & $264(35.4)$ & \\
\hline Cardiac Arrest & $252(2.9)$ & $197(2.5)$ & $55(7.4)$ & $<0.001$ \\
\hline Underwent $\mathrm{PCl}$ & $5560(79.3)$ & $5043(79.0)$ & $517(83.0)$ & 0.018 \\
\hline $\begin{array}{l}\text { Door to balloon time for STEMI } \\
\text { patients (min) }\end{array}$ & $87(406)$ & $81(321)$ & $140(855)$ & 0.172 \\
\hline Multivessel CAD & $62(1.0)$ & $51(0.9)$ & $11(1.9)$ & 0.018 \\
\hline Culprit vessel & & & & 0.002 \\
\hline Left Main & $136(2.0)$ & $116(1.9)$ & $20(3.4)$ & \\
\hline Left Anterior Descending & $3422(51.4)$ & $3086(50.9)$ & $336(56.6)$ & \\
\hline Circumflex & 766 (11.5) & $712(11.8)$ & $54(9.1)$ & \\
\hline Right Coronary Artery & $1817(27.3)$ & $1680(27.7)$ & $137(23.1)$ & \\
\hline Others & $512(7.7)$ & $465(7.7)$ & $47(7.9)$ & \\
\hline Number of stents & & & & 0.104 \\
\hline 1 & $3992(63.8)$ & $3610(63.5)$ & $382(67.5)$ & \\
\hline 2 & 1219 (19.5) & $1130(19.9)$ & 89 (15.7) & \\
\hline${ }^{3} 3$ & $335(5.4)$ & $307(5.4)$ & $28(4.9)$ & \\
\hline Number of vessels intervened & & & & 0.582 \\
\hline
\end{tabular}


3972 (88.3) $\quad 3591(88.4) \quad 381(87.0)$

Post-PCI TIMI

464 (10.3)

$415(10.2)$

49 (11.2)

$63(1.4)$

$55(1.4)$

$8(1.8)$

$$
0
$$

0

1

2

3

Procedural success

Coronary Artery Bypass Grafting

In-hospital complications

\begin{tabular}{|c|c|c|c|c|}
\hline Length of hospital stay (days) & $6.9(9.0)$ & $7.0(9.0)$ & $6.7(9.0)$ & 0.475 \\
\hline Sepsis & $561(6.6)$ & $520(6.7)$ & $41(5.6)$ & 0.265 \\
\hline New onset atrial fibrillation & $436(5.1)$ & $406(5.2)$ & $30(4.1)$ & 0.199 \\
\hline Major bleeding & $664(7.8)$ & $613(7.9)$ & $51(7.0)$ & 0.397 \\
\hline Inotropic support & $832(9.8)$ & $722(9.3)$ & $110(15.1)$ & $<0.001$ \\
\hline Intubation & $723(19.9)$ & $626(18.8)$ & 97 (31.7) & $<0.001$ \\
\hline Mitral regurgitation & $820(9.5)$ & $768(9.7)$ & $52(7.0)$ & 0.018 \\
\hline Ventricular arrhythmia & & & & 0.009 \\
\hline $\begin{array}{l}\text { Nonsustained ventricular } \\
\text { tachycardia }\end{array}$ & $413(11.5)$ & $370(11.2)$ & $43(14.9)$ & \\
\hline Sustained ventricular tachycardia & $188(5.2)$ & $165(5.0)$ & $23(8.0)$ & \\
\hline Acute renal failure & & & & 0.183 \\
\hline Not requiring dialysis & $828(21.5)$ & $774(21.8)$ & $54(18.2)$ & \\
\hline Requiring dialysis & $129(3.4)$ & $122(3.4)$ & $7(2.4)$ & \\
\hline \multicolumn{5}{|l|}{ Outcomes } \\
\hline All-cause mortality & $633(7.3)$ & $546(6.9)$ & $87(11.7)$ & $<0.001$ \\
\hline Cardiac related mortality & $564(6.5)$ & $484(6.1)$ & 80 (10.7) & $<0.001$ \\
\hline Cardiogenic shock & 669 (9.3) & 574 (8.6) & 95 (16.3) & $<0.001$ \\
\hline Stroke & $167(2.0)$ & 150 (1.9) & $17(2.4)$ & 0.396 \\
\hline
\end{tabular}


CAD - Coronary Artery Disease; ACEI - Angiotensin Converting Enzyme Inhibitor; ARB - Angiotensin II Receptor Blocker; ACS - Acute Coronary Syndrome; PCI - Percutaneous Coronary Intervention; STEMI ST Elevated Myocardial Infarction; NSTEMI - Non-ST Elevated Myocardial Infarction; TIMI Thrombolysis in Myocardial Infarction

* Categorical variables are presented as $\mathrm{n}(\%)$ and continuous variables are presented as Mean (Standard Deviation)

\section{Figures}

\section{Prevalence of SMuRF-less population over time}

1200

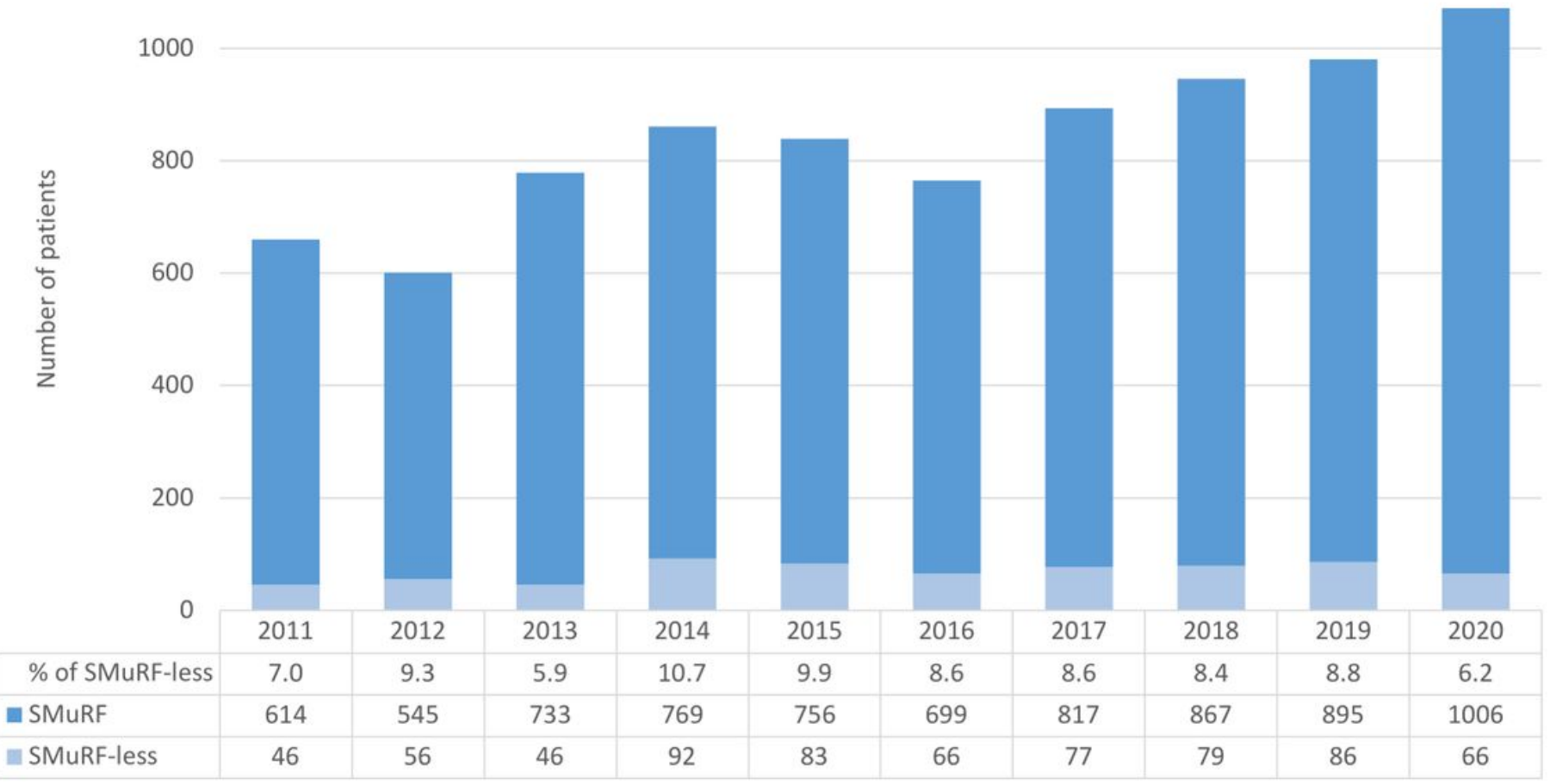

\section{Figure 1}

Bar graph displaying the prevalence of the SMuRF-less population from 2011 to 2020. 

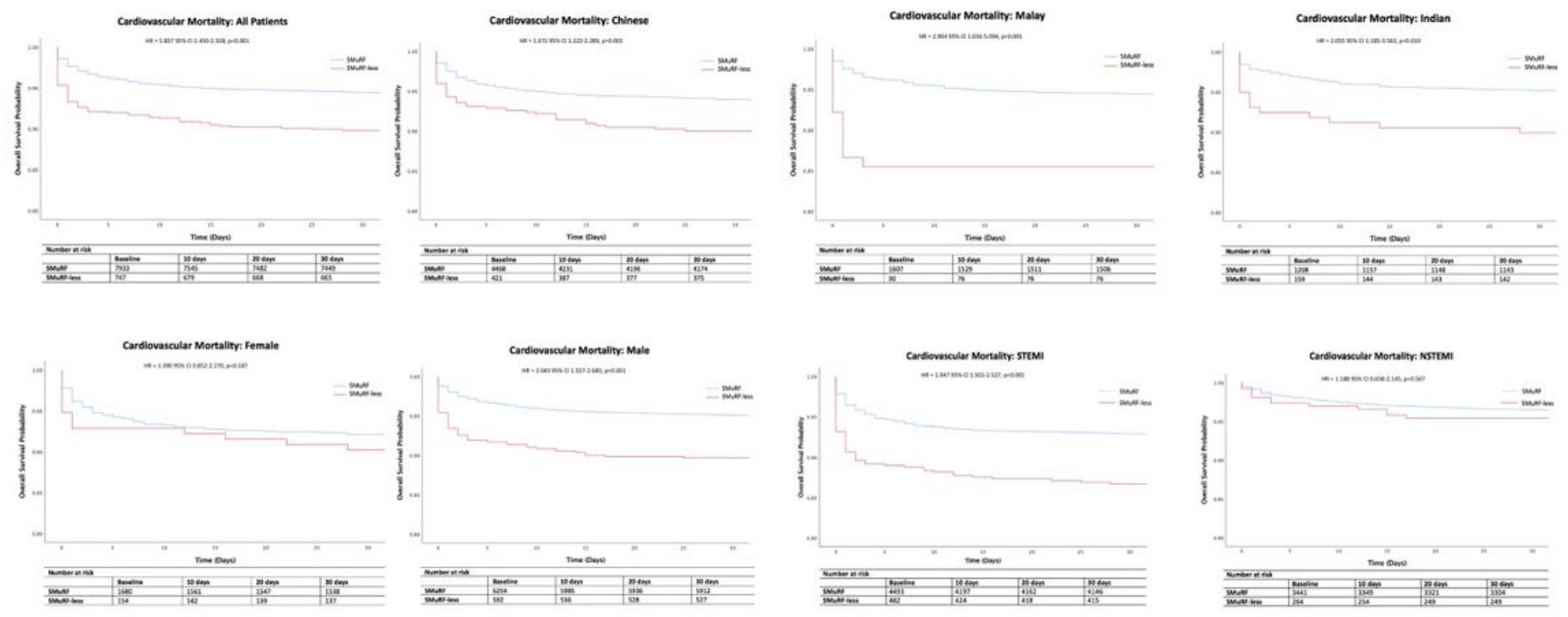

Figure 2

Kaplan Meier curves for 30-day cardiovascular mortality in all patients presenting with acute coronary syndrome, with subgroup analysis based on sex, ethnicity and ACS type. 


\section{Unadjusted}

Cardiovascular Mortality (HR)

30-Day Readmission (RR)

Cardiogenic Shock (RR)

Stroke (RR)

Heart Failure (RR)

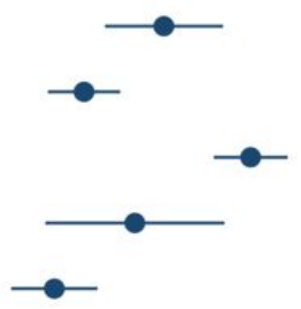

$1.40[1.08,1.73]$

$0.95[0.76,1.15]$

$1.89[1.69,2.09]$

$1.24[0.74,1.74]$

$0.79[0.55,1.02]$

$1.26[1.16,1.37]$

\section{Adjusted}

Cardiovascular Mortality (HR)

30-Day Readmission (RR)

Cardiogenic Shock (RR)

Stroke (RR)

Heart Failure (RR)

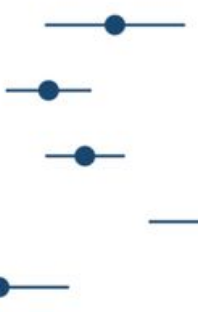

$1.48[1.09,1.86]$

$1.10[0.87,1.34]$

$1.31[1.09,1.52]$

2.51 [ $1.67,3.34]$

0.82 [ $0.44,1.21]$

$1.23[1.10,1.37]$

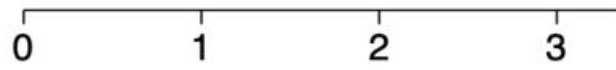

\section{Figure 3}

Forest plot comparing unadjusted and adjusted study outcomes in SMuRF-less and SMuRF patients presenting with acute coronary syndrome. 